\title{
Une étude de cas : le patrimoine juif dans les pays de l'ex-Yougoslavie
}

A Case Study: Jewish Patrimony in Former Yugoslavia Countries

Ivan Ceresnjes

\section{(2) OpenEdition}

Journals

Édition électronique

URL : http://journals.openedition.org/etudesbalkaniques/81

ISSN : 2102-5525

\section{Éditeur}

Association Pierre Belon

\section{Édition imprimée}

Date de publication : 1 janvier 2005

Pagination : 211-218

ISBN : 2-910860-05-1

ISSN : $1260-2116$

\section{Référence électronique}

Ivan Ceresnjes, «Une étude de cas : le patrimoine juif dans les pays de l'ex-Yougoslavie ", Études balkaniques [En ligne], 12 | 2005, mis en ligne le 07 avril 2009, consulté le 19 avril 2019. URL : http:// journals.openedition.org/etudesbalkaniques/81

Ce document a été généré automatiquement le 19 avril 2019

Tous droits réservés 


\title{
Une étude de cas : le patrimoine juif dans les pays de l'ex-Yougoslavie
}

\author{
A Case Study: Jewish Patrimony in Former Yugoslavia Countries
}

Ivan Ceresnjes

1 La culture unique des Juifs de l'ex-Yougoslavie n'a jamais été étudiée de façon systématique. En raison des ravages de l'Holocauste et des effets des récents conflits internes, il reste moins de 5.700 Juifs sur les 85.000 présents avant la Seconde Guerre mondiale. Comme l'ensemble du pays, la communauté juive se définissait autrefois par une combinaison unique de traditions orientales et occidentales. Des populations importantes de juifs sépharades et ashkénazes coexistaient, dans des villes comme Sarajevo, avec leurs voisins chrétiens et musulmans. En 1988, le Center of Jewish Art (The Hebrew University, Jerusalem) lança un projet majeur pour inventorier les restes matériels du patrimoine culturel juif de l'ancienne Yougoslavie. Le projet fut interrompu par la guerre civile qui fit rage entre 1991 et 1995 et ne reprit que dix ans plus tard. Les premières campagnes se concentrèrent sur l'inventaire des objets de culte conservés dans les principales villes, Zagreb, Split, Dubrovnik, Sarajevo et Belgrade.

2 Depuis 1988, le Centre a fait porter ses efforts sur les monuments en péril du patrimoine juif, spécialement dans les régions dévastées par la guerre civile. Au cours de cinq campagnes, le Centre a inventorié quarante-sept synagogues et salles de prière, quinze chapelles de cimetières sur trente, sept mausolées sur vingt-et-un; il a prospecté cent cinquante cinq cimetières sur deux cent cinquante cinq ainsi que huit musées conservant de petites collections d'objets rituels et de dalles funéraires médiévales. Une prospection a également été effectuée dans les édifices de diverses institutions juives, telles que les écoles, les maisons rabbiniques, les boucheries rituelles.

3 Bien que les Juifs n'aient pas été directement visés par la récente guerre civile, ils ont néanmoins été sérieusement affectés par le conflit qui a conduit beaucoup d'entre eux à abandonner leur maison; à l'exception d'un petit nombre de cas, la condition des sites juifs est désespérée. Souvent nationalisées ou confisquées par le précédent régime, les 
synagogues tombent en ruine l'une après l'autre. Certaines ont été transformées de manière à les rendre méconnaissables en ensembles d'appartements, en usines, en ateliers ou en magasins tandis que d'autres conservent un usage cultuel et sont de ce fait relativement bien entretenues. Le sort des cimetières est encore plus sombre. Le Centre a découvert des cimetières juifs oubliés, sur les pentes de collines dominant les lieux où vécurent autrefois ceux qui y sont ensevelis mais où, aujourd'hui, nul ne se souvient d'eux. Les chercheurs du Centre ont également identifié des cimetières dans de petites villes, voire dans des villages où ne survivent qu'un ou deux juifs : ceux-ci se demandent avec angoisse qui prendra soin du cimetière après leur mort.

4 Cet article fera connaitre quelques exemples du riche patrimoine juif de l'exYougoslavie inventoriés par le Center for Jewish Art, se bornant à signaler les petites collections d'objets rituels de Bjelovar, Koprivnica, Travnik et Zenica ainsi que les deux importantes collections du Centre communautaire juif et du musée des arts et traditions de Zagreb qui ont été recensées au même titre que la collection de peintures dues à des artistes juifs conservée au Musée juif de Belgrade.

\section{Les synagogues}

5 Sur le territoire de l'ex-Yougoslavie, des synagogues existèrent dès le second et le troisième siècle, mais seuls les vestiges de deux anciennes synagogues subsistent à Salona (Croatie) et Stobi (Macédoine). On a également découvert quelques inscriptions lapidaires qui suggèrent l'existence de proseuche (expression grecque synonyme de synagogue) à partir du quatrième siècle à Mursa (près d'Osijek en Croatie) et à Dabravina (près de Zenica en Bosnie-Herzégovine) où la synagogue serait d'époque romaine ou byzantine.

6 Le seul édifice subsistant de la période médiévale est la synagogue de Maribor (Slovénie), édifiée entre le $\mathrm{XII}^{\mathrm{e}}$ et le $\mathrm{XV}^{\mathrm{e}}$ siècle. Remontant aux débuts de la domination ottomane sur la Bosnie, le Vieux Temple (Il Kal Vijezu) de Sarajevo est le témoin suivant. Viennent ensuite deux synagogues baroques de Croatie, l'une à Dubrovnik, l'autre à Split qui comptent parmi les meilleurs exemples de la transposition d'édifices civils en édifices religieux par l'architecture juive ; les seules synagogues conservées du XVIII siècle sont celles de Lendava (Slovénie) et du Nouveau Temple (Il Kal Muevu) de Sarajevo (BosnieHerzégovine).

7 Que ce soit par suite des guerres, des épidémies, de l'instabilité politique, de l'antisémitisme sous diverses formes, on observe un important hiatus chronologique dans la construction des synagogues, grosso modo entre le XVII ${ }^{e}$ et le début du XIX siècle. Depuis lors, pourtant, de nombreux bâtiments importants -témoins de tous les styles architecturaux, de l'historicisme au Bauhaus- ont été construits.

8 Pour la période de l'historicisme on peut distinguer quatre types fondamentaux de structures. Ils ne diffèrent pas tant par l'aménagement intérieur que par leur apparence extérieure et la manière dont ils s'insèrent dans le paysage urbain.

\section{La synagogue-maison}

9 Bien que conçue en tant qu'édifice religieux, elle suit clairement la tradition des premières maisons de prière. Ce type de synagogue est identique à celui d'un des bâtiments à un ou deux étages de son environnement; il comporte souvent un mur- 
pignon triangulaire et un toit en bâtière. Les portes et les fenêtres ressemblent à celles des bâtiments voisins et les dimensions s'accordent également avec celles des autres constructions de la rue. La synagogue-maison du XIX siècle se réfère à des structures médiévales, comme celle de l'Altneuschul de Prague (1280) ou celles des modèles originaux des synagogues de Split et Dubrovnik en Croatie, de Maribor en Slovénie.

10 Il existe neuf exemples de synagogue-maison au XIXe siècle si l'on tient compte de la première petite synagogue de Bjelovar en Croatie (bâtie en 1882 mais aujourd'hui détruite) et de celle de Podravska Slatina, également en Croatie (1896-1900). Au début et à la fin de la période où furent construites les synagogues de Croatie, il y eut des variantes monumentales de ce type de synagogue de grandes dimensions et libres de toute construction mitoyenne. Ainsi, par exemple, celle de Čakovec (1836), qui ressemble à la vieille mosquée sépharade de Belgrade.

11 Le bâtiment moderne de la synagogue de Niš (Serbie-Monténégro) variante du "style Sécession » (1926) est un exemple exceptionnel. En Bosnie, les synagogues de Travnik, Rogatica, la Nouvelle Synagogue et la Bjelave Synagogue, peuvent également être inscrites dans ce groupe, en complément à la vieille synagogue de Skopje et à cinq autres construites à Bitola (Macédoine) et détruites à l'issue de la seconde guerre mondiale.

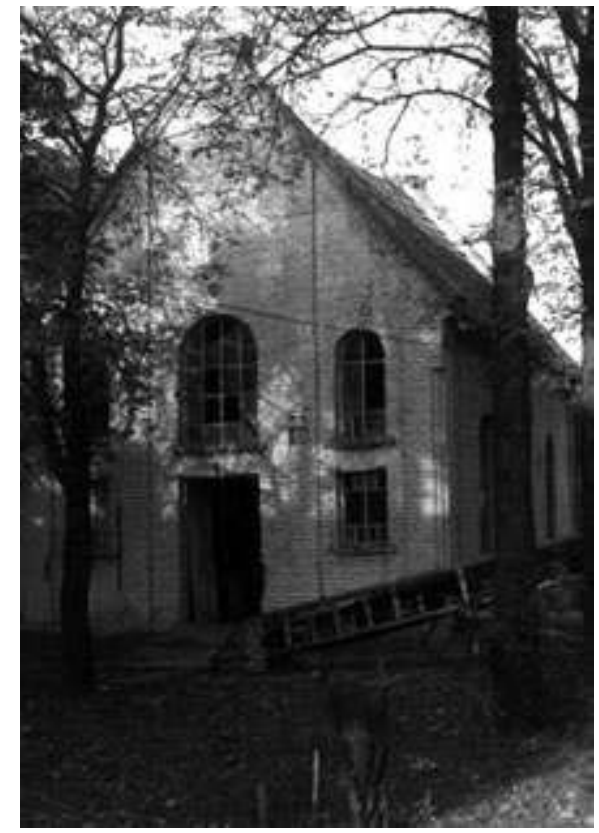

Ancienne synagogue à Hajdukovo, Vojvodine, 1895

\section{La synagogue tripartite}

12 Elle se développa à partir du modèle créé par l'architecte viennois Ludwig von Förster dont les plans pour les lieux de culte des Néologues (Juifs réformés) eurent une influence considérable sur l'architecture des synagogues dans tout l'Empire austro-hongrois.

13 La façade principale de ces synagogues se divise en trois sections verticales, la section axiale étant généralement plus élevée que les sections latérales. Le schéma suggère un plan basilical avec nef et bas-côtés.

14 Ce modèle de synagogue, avec son plan rectangulaire, était considéré comme idéal pour les synagogues réformées où l'on avait abandonné l'organisation bipolaire de 
l'espace. Dans les premières synagogues ashkénazes, l'arche de la Torah ou armoire de la Loi était située sur le mur oriental et le bimah, où la Torah était lue, placé dans l'espace de prière central. Dans les synagogues sépharades, le bimah fut repoussé vers le mur opposé, du côté ouest de la synagogue. Dans les synagogues réformées du XIXe siècle, toutefois, cette bipolarité fut abandonnée, et le bimah directement placé face à l'arche de la Torah.

15 La synagogue de Zagreb (1866-1867), œuvre de l'architecte Franjo Klein, était un prototype de ce groupe. Citons également celle de Koprivnica (1875-1876) en Croatie, plus modeste et provinciale. Parmi les huit exemples de synagogues tripartites connues dans ce pays, certaines déviations et atténuations du schéma directeur de la façade existent, mais l'accent est toujours mis sur la surélévation du corps central, comme à Virovitica (1860-1863) et à Križevci (1895). Les exemples typiques en Serbie étaient la synagogue sépharade de Zemun et la synagogue de Sremska Mitrovica, toutes deux détruites au cours de la seconde guerre mondiale. La synagogue néo-mauresque de Zenica (BosnieHerzégovine), bâtie en 1903, reflète également le même concept architectural.

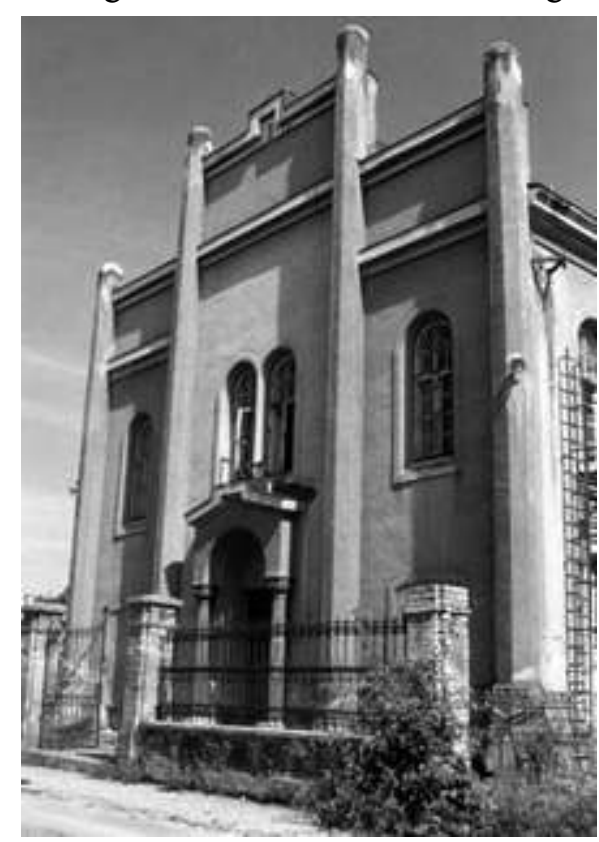

La synagogue à Koprivnica,1876

\section{La synagogue à tours jumelles}

16 Imitant l'organisation des façades d'église, avec leurs deux clochers latéraux, ce type convient à un environnement urbain chrétien, mais apparaît aussi dans des contextes environnementaux hétérogènes, reflétant l'influence des styles contemporains. Les plans de Ludwig von Förster pour la synagogue réformée de Budapest (1854-1859) sont à l'origine de ce type. Les synagogues croates qui s'y conformèrent eurent généralement des tours beaucoup plus petites, retenant seulement une partie octogonale surmontée par un dôme en forme de bulbe, des couronnements d'apparence « exotique » distinguaient la silhouette des synagogues de celle des églises catholiques.

17 En Croatie on construit dix synagogues de ce type: la synagogue de Varaždin (1860-1861), la chapelle du cimetière de Djakovo (1879) et la belle synagogue «Sécessionniste» de Bjelovar (1912-1914). Beaucoup de synagogues de ce type furent 
bâties en Serbie, dans des agglomérations de Vojvodine, à la suite de la synagogue sépharade de Belgrade, détruite en 1941.

18 Ce type de synagogue était courant dans la Bosnie annexée à l'Empire austrohongrois: par exemple Banja Luka (1902), Bijeljina (1895), Jajce, Derventa, Zvornik, Višegrad, Brčko et autres localités. La synagogue ashkénaze néo-mauresque de Sarajevo, construite en 1902 par Karl Pařik, se rattache également au même type, bien qu'elle ait quatre tours, et non deux.

\section{La synagogue à coupole}

19 Ce type, le moins répandu en Croatie, apparut dans ce pays d'après des modèles conçus à Vienne et en Hongrie à l'époque où l'historicisme était à son apogée. Bien qu'en Europe nombre d'importantes synagogues à coupole aient été construites -comme par exemple le Tempio Maggiore de Florence en 1874-1882-, ce type reste plus proche du style sépharade, avec un riche décor mauresque et oriental, que des synagogues ashkénazes de l'intérieur du pays. Dans les villes de Croatie où les communautés juives étaient disposées à accepter le style hongrois, des synagogues à coupole furent construites par Lippot Baumhorn à Vukovar (1889), Rijeka (1902-1903) et Vinkovci (1922-1923), incorporant des éléments issus d'un académisme ultérieur. L'importante chapelle du cimetière juif de Vukovar appartient clairement à ce groupe, avec son aspect historiciste attardé et les éléments « Sécession» du décor chrétien. Selon le premier guide de Zagreb rédigé par A. Hudovski en 1892, la synagogue de la rue Praska «... reste inachevée, car le dôme surmontant la façade manque ».

20 En Serbie, dans la région de Vojvodine, les plus célèbres exemples de ce type furent bâtis à Subotica (1902) par Komor et Jakab, à Novi Sad (1909) et Zrenjanin (Nagy Becskerek, 1896) par Lippot Baumhorn, sans compter de plus petites synagogues en milieu rural.

21 La seule synagogue à coupole de Bosnie fut construite à Sarajevo en 1926 par Rudolf Lubinski, en style néo-byzantin ${ }^{1}$. 


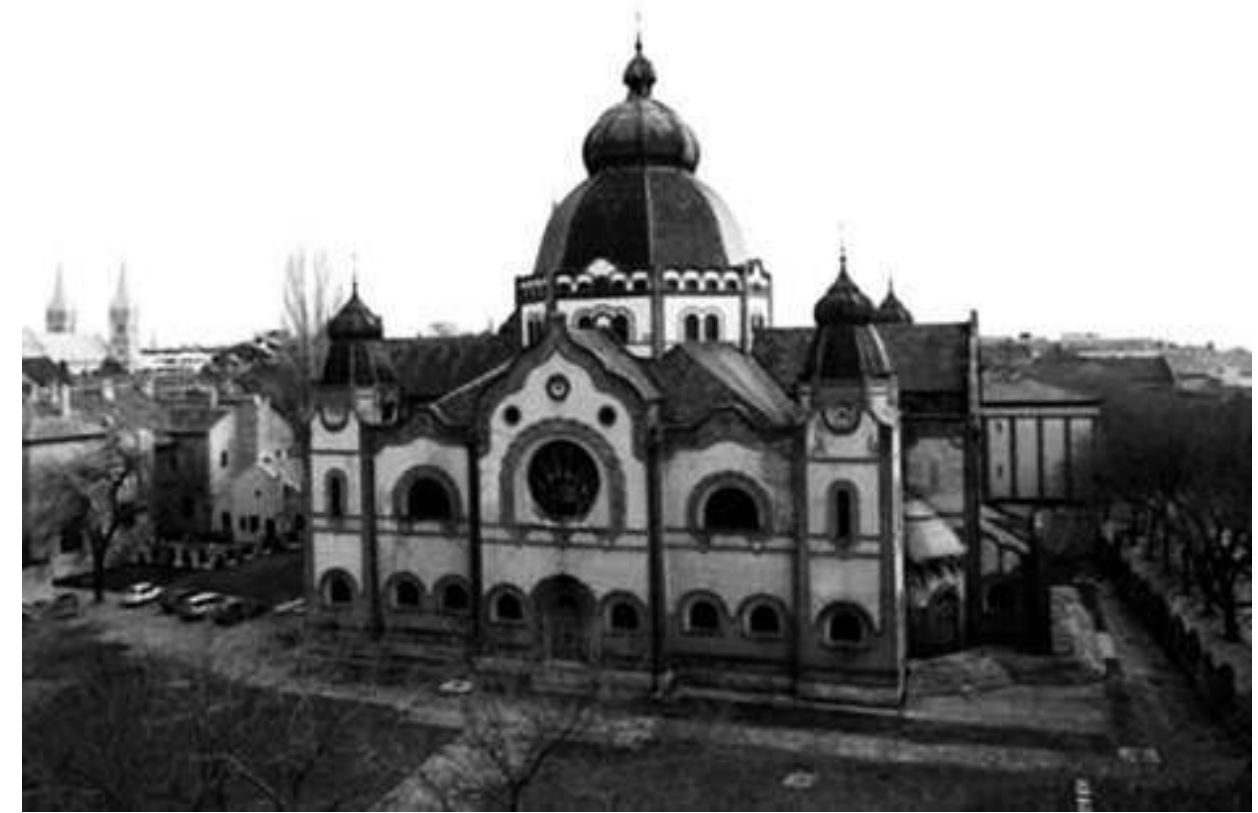

Ancienne synagogue à Subotica, Vojvodine, 1902

\section{Les cimetières}

22 Les cent trente communautés juives présentes dans les pays de l'ex-Yougoslavie avaient environ deux cent quatre vingt dix cimetières. Il est difficile d'établir une typologie claire des monuments funéraires, compte tenu des différentes appartenances communautaires, de la diversité des situations historiques dans des régions où coutumes funéraires et traditions juives s'exprimaient de façon variée. En général, cependant, on peut distinguer quatre groupes.

- Les anciennes sépultures sépharades dans les zones influencées par la tradition catholique sont des dalles funéraires horizontales ou des sarcophages de faible hauteur. D'abord tracées en hébreu, les inscriptions comportèrent ensuite des additions en langue locale.

- Les anciennes sépultures sépharades dans les zones sous domination ottomane où les Juifs suivaient la coutume de leur précédente patrie, influencée par les traditions pré-islamiques locales dites «Bogomiles». Ce sont des monuments monolithes coiffés d'un berceau. Les inscriptions sont en hébreu, rarement en ladino et en caractères hébreux. Elles comportent plus tard des additions en langue locale.

- Les anciennes sépultures ashkénazes en forme de stèles verticales en pierre. Celles des rabbins ashkénazes combinent parfois stèle et sarcophage. Les inscriptions sont gravées soit en hébreu, soit en yiddish et en caractères hébreux.

- À partir du milieu du XIX ${ }^{\mathrm{e}}$ siècle les monuments funéraires des juifs de toutes dénominations ressemblent souvent à ceux des chrétiens, qu'il s'agisse d'obélisques de marbre, d'édifices de formes variées, pouvant même comporter des sculptures et des portraits des défunts. Les inscriptions sont en allemand, en hongrois, ou dans l'une des langues des Slaves du Sud, avec parfois de l'hébreu.

23 Dans des lieux habités par une majorité de catholiques, des fragments de monuments funéraires juifs ont été réemployés comme matériaux de construction pour paver des rues ou réparer des murailles urbaines. Des recherches ont révélé il y a peu le réemploi de 
tombes des XVI ${ }^{e}$ et XVII ${ }^{e}$ siècles dans le couronnement des fortifications de Dubrovnik. De plus, on a identifié des pierres tombales d'époque médiévale dans les collections lapidaires de Ptuj et de Maribor, en Slovénie. Récemment encore, la pierre tombale d'un marchand juif de la fin du XVe siècle a été découverte en Bosnie centrale ; elle porte une inscription rédigée en caractères glagolitiques dans la langue locale.

\section{NOTES}

1. Cette division typologique en quatre groupes n'est pas absolue: certaines synagogues y trouvent leur place, d'autres non. Néanmoins, la classification offre une base opérationnelle à la poursuite des recherches menées par le Centre sur le patrimoine architectural de l'exYougoslavie.

\section{RÉSUMÉS}

L'auteur se propose de palier le manque d'une étude de la "culture unique » des Juifs de l'exYougoslavie, en brossant rapidement son tableau. Il appuie sa démarche sur le travail lancé en 1988 par le Centre d'Art juif (Hebrew University, Jérusalem) pour inventorier les restes matériels de ce patrimoine. Sont évoqués les diverses typologies de synagogues, ainsi que les cimetières.

The author's task is to offset the lack of a study on the cultural unity of Jews in former Yugoslavia. He uses the program launched by the Center for Jewish Art (Hebrew University of Jerusalem) in 1988 to make a list of what concretely remains of this patrimony. Here are analyzed the diverse typologies of synagogues, as well as cemeteries.

\section{AUTEUR}

\section{IVAN CERESNJES}

Université hébraïque, Jérusalem 